\title{
GAP ACCEPTANCE DECISION MODEL FOR U-TURN MOVEMENT AT MIDBLOCK MEDIAN OPENING
}

\author{
Thakonlaphat JENJIWATTANAKUL 1 and Kazushi SANO ${ }^{2}$ \\ ${ }^{1}$ Graduate Student, Dept. of Civil and Environmental Eng., Nagaoka University of Technology \\ (1603-1 Kamitomioka, Nagaoka, Niigata 940-2188, Japan) \\ E-mail: s107017@stn.nagaokaut.ac.jp \\ ${ }^{2}$ Associate Professor, Dept. of Civil and Environmental Eng., Nagaoka University of Technology \\ (1603-1 Kamitomioka, Nagaoka, Niigata 940-2188, Japan) \\ E-mail: sano@nagaokaut.ac.jp
}

\begin{abstract}
U-turn movement at the midblock median opening is primarily based on gap acceptance process. This research investigated the factors affecting the u-turn decision of the drivers and evaluated the statistical significance and influence levels of each factor. The u-turn decision prediction model had been developed after finalizing the significant factors. The field data was collected at $3 \mathrm{u}$-turn locations on an urban arterial in Bangkok, Thailand. The u-turning vehicles in the study included car, taxi, and pick-up, which the passenger car equivalent (PCE) equal to 1 . The binary logistic regression technique was employed in the data analysis and model development. From the considered eight factors, gap size, speed of conflicting vehicle, and wait time at the front position of the queue were statistically significant at the confidence interval of $95 \%$. In this study, the effects of wait time and queue time were separately examined. It was interesting to found that the queue time did not significantly affect the u-turn decision. The developed decision model, which explanatory variables included gap size, conflicting speed, and wait time, could predict the u-turn decision well with the percentage correctness of more than $85 \%$.
\end{abstract}

Key Words : u-turn, gap acceptance, logistic regression, decision model

\section{INTRODUCTION}

U-turn movement at an unsignalized midblock median opening is based on the gap acceptance behavior. When a vehicle arrives at a median opening, its driver faces the gaps of the conflicting through traffic, waits for an acceptable gap, and then makes a u-turn. Since the u-turn movement is complex and may lead to safety concerns, the factors affecting the u-turn decision were investigated in this study.

The objectives of this research were as follows:

(i) to evaluate the factors affecting the u-turn decision in terms of their statistical significance and level of influence;

(ii) to develop a u-turn decision model, in order to predict the decision under variety of factors.

To improve the traffic control strategies at or near the u-turn junctions, knowledge of affecting factors is essential. The developed model can be used to find some control criteria or threshold values. The model can also be used in the simulation modeling.
Eight factors were considered; five relating to the u-turning vehicle (driver age, gender, vehicle type, queue time, and wait time), the other three relating to the conflicting traffic (gap size, speed, and vehicle type). The binary logistic regression technique was employed in the data analysis process. The analysis was conducted in an aggregate manner to represent the overall behavior of u-turning vehicles. It was not site specific or time specific; the decision was based on the conditions that the driver faced.

The results showed that those factors had effect on the u-turn decision in the following descending order: gap size, conflicting speed, wait time, conflicting vehicle type, gender, age, u-turning vehicle type, and queue time. The significant factors included gap size, conflicting speed, and wait time at the confidence interval of $95 \%$. The combination of u-turning and conflicting vehicle type was also examined and found statistically insignificant. It could be noticeable that the queue time of the u-turning vehicle did not significantly affect the gap acceptance decision, 
but the wait time did.

The u-turning vehicles in this study focused only on passenger car category, which included all kinds of vehicle with passenger car equivalent (PCE) of 1 . This included private car, taxi, and pick-up truck. The conflicting vehicle also include heavy vehicles (bus and large truck).

\section{LITERATURE REVIEW}

The gap acceptance process is the theoretical basis for the methodology of capacity analysis of a two-way stop-controlled (TWSC) intersection ${ }^{1)}$. The capacity estimation methods providing for TWSC intersection can be applied to estimating u-turn capacity at unsignalized median openings ${ }^{2}$. Therefore, it can imply that the behavior of the u-turning vehicle is similar to that of the minor-street vehicle at the TWSC intersection.

The different conditions (combination of driver, vehicle, traffic, and environment conditions) result in the different gap acceptance behavior at TWSC intersections. For example, the difference in driver age group affects the gap acceptance capabilities ${ }^{3)}$. Regarding gender, the male drivers tend to accept smaller gaps than female drivers ${ }^{4}$. In addition, the mean accepted gap tends to decrease as the queue time or wait time increases ${ }^{5}$.

A decision model for gap acceptance at intersections has been developed based on a risk-reward loop process. The result shows that the entry onto the main road occurs when the benefit from entry is greater than the associated risk ${ }^{6}$.

U-turns are widely used in many countries as the alternative to direct left-turns (driving on the right rule) at busy intersections. The u-turn traffic flow model, applying cellular automata simulation, has been developed to demonstrate the traffic flow condition at a u-turn junction ${ }^{7}$. The result shows that the effectiveness of u-turn facility is limited to low traffic density and minimal lane changing maneuvers. A new u-turn design with protected u-turn movement has been proposed and compared, in term of travel time, with conventional signalized intersection ${ }^{8}$. The comparison shows that this new u-turn facility generally produces lower travel time, especially when the traffic flow is high.

There were only a few studies on gap acceptance characteristics of u-turn junction. One study shows that the combination of gap size and acceleration of priority vehicle gives the best and most consistent definition of the probability of gap acceptance of u-turning vehicles ${ }^{9}$. The speed of the priority vehicle and wait time of u-turning vehicle are among the insignificant factors. However, the result is based on the data collected at one site on one day. The presence of crosswalk and minor road's merging flow may affect the u-turn gap acceptance characteristics. Another study focuses on the aggressive driver behavior. In the gap acceptance process, there might be some aggressive drivers who force their vehicles into the major stream, making the conflicting vehicles to slow down or stop. Three major factors, affecting the probability of such a forcing maneuver, include driver age, car performance, and average speed on the major road ${ }^{10)}$. The driver's total waiting time, while waiting for an acceptable gap, is of little significance in incurring the forcing behavior. A recent study investigates the effect of waiting time on gap acceptance behavior and found that the larger waiting time, the smaller accepted gap ${ }^{11)}$. The more waiting time would frustrate the drivers to accept the significant smaller gap, leading to the aggressive behavior. In this present research, all the relevant factors to the u-turn decision were taken into consideration. The waiting time was reinvestigated herein to check its significance.

\section{METHODOLOGY}

The gap acceptance decision is the dichotomous problem, of which the possible solution will be either "yes" or "no". The binary logistic regression analysis is widely used to model the occurrence of such an event. The output of the model is the probability of the event occurrence. In addition, the logistic regression also gives the statistical significance level of each variable.

The probability of the u-turn decision based on the explanatory variables $x_{1}, x_{2}, \ldots, x_{n}$ can be modeled as:

$$
\begin{aligned}
& \mathrm{P}(\text { accept })=\frac{e^{z}}{1+e^{z}}=\frac{1}{1+e^{-z}} \\
& z=\beta_{0}+\beta_{1} x_{1}+\beta_{2} x_{2}+\ldots+\beta_{n} x_{n}
\end{aligned}
$$

where $\beta_{0}, \beta_{1}, \beta_{2}, \ldots, \beta_{n}$ are the parameters estimating from the logistic regression analysis.

The analysis started with all variables to determine the significance of each variable. The forward and backward stepwise analyses, based on likelihood ratio, were also conducted to validate the level of influence of variables. The level of significance was set at 0.05 for the variable entry and 0.10 for the variable removal in the stepwise analysis. The forward stepwise analysis results were used to evaluate the effect of significant variables. The cutting value for the decision of accepting the gap was set at the probability of 0.5 . 


\section{DATA COLLECTION}

\section{(1) Site characteristics}

To get sufficient and various samples, three midblock median openings were selected for data collection. Those sites are located on a six-lane divided street with three lanes in each direction as shown in Fig.1. The physical geometry characteristics of the 3 sites are similar. There is an exclusive u-turn bay for both directions at all sites. Most u-turning vehicles encroach to the middle lane when they make a u-turn. Therefore, the vehicles on the middle and median lanes were treated as the conflicting vehicles. The analyses were conducted in an aggregate manner so that the results could be applied in general, not site specific.

\section{(2) Considered factors}

The factors that can be simply measured and realized in the field were considered in this study. This would demonstrate and represent the decision of the drivers of u-turning vehicles. The acceleration of the conflicting vehicle was not included because it is very difficult to be recognized by the u-turning vehicle when making a decision.

Eight variables were included in this study:

(i) u-turn driver age group, dividing into three groups as young, middle, and old.

(ii) u-turn driver gender.

(iii) u-turning vehicle type, dividing into car (sedan, sport utility vehicle, and van), taxi, and pick-up.

(iv) queue time, which is the time duration that the u-turning vehicle starts to join the queue until it moves to the front of the queue.

(v) wait time at the front position of the queue. (vi) gap size, referring to time headway of conflicting traffic in this study. This is to facilitate the data collection. As changes in the latest Highway Capacity Manual 2010, the word "headway" replaces "gap" for the parameters of gap acceptance to estimate the potential capacity of TWSC intersections ${ }^{1)}$.

(vii) speed of the conflicting vehicle.

(viii) conflicting vehicle type, dividing into car (sedan, sport utility vehicle, and van), taxi, pick-up, and heavy vehicle (bus and large truck).

The variables (i) - (v) related to the u-turn traffic while the remaining related to the conflicting traffic. In addition, the combination of u-turning vehicle type (iii) and conflicting vehicle type (viii) were also of interest. It is assumed that all u-turning vehicles have similar capability to recognize gap and speed of conflicting vehicles.

\section{(3) Field data collection}

The data were collected for two days at each site. The collection periods on each day included morning peak (07:00-09:00), off-peak (11:00-13:00), and evening peak (16:00-18:00) in order to get sufficient sample data and cover most ranges of traffic conditions. The data from all periods were combined for analysis to get the average behavior of all u-turning vehicle samples.

Two kinds of data were collected in the field, i.e. video data and sound data. A video camera was set at the nearby pedestrian bridge to record the traffic movement. The video data was reviewed to collect useful information in the laboratory, i.e. vehicle type, queue time, wait time, gap size, and speed. Since the instantaneous speed data was not available, the speed in this study was calculated based on the travel time between a known fixed distance at each site.

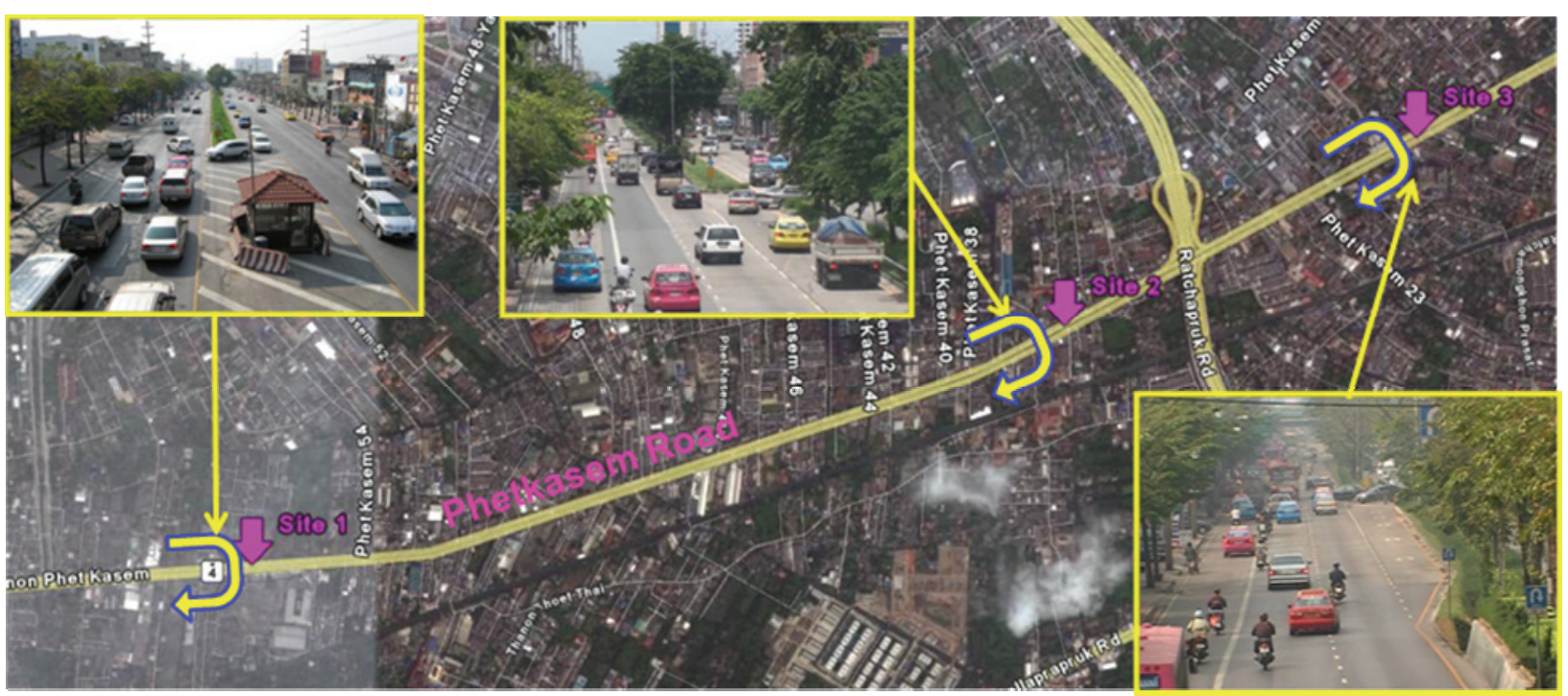

Fig. 1 Site location and traffic condition. 
The sound data was recorded by another observer, who was near the u-turn location. The observer recorded the u-turn driver age and gender of some random vehicles. The follow-up u-turning vehicles were not considered because they did not perform the real gap acceptance behavior. Since the driver age information was based on the observer's perception, the age data was divided into rough three groups to minimize the human error.

\section{(4) Data processing}

The recorded data were reviewed to extract the usable data and information. The data, which did not represent the pure gap acceptance behavior of u-turn, were not used in the analysis. For example, the data during the following situations could not be used:

- the conflicting traffic was congested;

- the conflcting vehicle stopped for u-turn; and

- the u-turn junction was controlled by police.

After the data reviewing, a total of $337 \mathrm{u}$-turning vehicles were extracted from the sound and video data. Each vehicle faced one or many rejected gaps and accepted only one gap. The gap size was known as the most influential factor of gap acceptance process. Definitely, the driver would reject small gaps and accept large enough gap. However, $22 \mathrm{u}$-turning vehicles accepted the gap smaller than their maximum rejected gap. This analysis was selective in order to explain the reasons why a driver rejected a large gap or accepted a small gap. To weight each driver the same, a pair of largest rejected gap and accepted gap were included in the analysis. Therefore, 674 cases were collected (337 vehicles, 2 gaps each). The conflicting speed data was collected after identifying the vehicles of interest.

The cases with gap less than or equal 2 seconds were screened out since no u-turning vehicle could take such a small headway of conflicting vehicles for u-turn maneuver. On the other end, the cases with gap more than or equal 10 seconds were also screened out because all u-turning vehicles could take such a large gap. As a result, the further analysis included a total of 593 cases of the u-turn decision, of which the random 530 cases were used for model development and the remaining 63 cases were used for model evaluation.

\section{RESULTS}

\section{(1) Correlation analysis}

From all 593 data cases, the correlation analysis was conducted and the Pearson correlation coefficient was shown in Table 1. It could be noticed that the u-turn decision had a high correlation with gap size. The correlations with conflicting speed and wait time were in the fair level. The result indicated a potential relationship among u-turn decision, gap size, conflicting speed, and wait time.

\section{(2) Variable selection}

The logistic regression analysis was conducted by entering all considered variables in order to evaluate their significance levels. The $\mathrm{p}$-value of each variable was shown in Table 2. When the p-value is less than the preset significance level, the null hypothesis is rejected. The result is said to be statistically significant. In the analysis, the null hypothesis is no difference on having the variable in model. On the other words, the variable with higher p-value has less effect on the u-turn decision.

The influence of variables to the decision in the descending order was as following: gap size $>$ conflicting speed $>$ wait time $>$ conflicting vehicle type $>$ combination of vehicle type $>$ gender $>$ age $>$ u-turning vehicle type $>$ queue time. Only three variables were statistically significant, at the significance level of 0.05 , including gap size, conflicting speed, and wait time. The combination of u-turning and conflicting vehicle type was also insignificant.

Table 1 Pearson correlation coefficient between all variables.

\begin{tabular}{|l|c|c|c|c|c|c|c|c|}
\hline \multicolumn{1}{|c|}{ Variables } & Gender & Age & $\begin{array}{c}\text { U-turn } \\
\text { Vehicle } \\
\text { Type }\end{array}$ & $\begin{array}{c}\text { Queue } \\
\text { Time }\end{array}$ & $\begin{array}{c}\text { Wait } \\
\text { Time }\end{array}$ & Gap Size & $\begin{array}{c}\text { Conflicting } \\
\text { Speed }\end{array}$ & $\begin{array}{c}\text { Conflicting } \\
\text { Vehicle } \\
\text { Type }\end{array}$ \\
\hline U-turn Decision & -0.02 & 0.00 & 0.00 & -0.03 & $\mathbf{0 . 3 5}$ & $\mathbf{0 . 6 9}$ & $\mathbf{- 0 . 3 6}$ & -0.03 \\
\hline Gender & & -0.06 & -0.18 & -0.04 & 0.03 & 0.02 & -0.02 & 0.01 \\
\hline Age & & & 0.05 & 0.05 & -0.07 & 0.05 & 0.10 & -0.07 \\
\hline U-turn Vehicle Type & & & & -0.07 & -0.02 & 0.02 & 0.03 & 0.03 \\
\hline Queue Time & & & & & -0.03 & -0.01 & 0.12 & 0.01 \\
\hline Wait Time & & & & & & $\mathbf{0 . 2 3}$ & $\mathbf{- 0 . 2 3}$ & 0.06 \\
\hline Gap Size & & & & & & & $\mathbf{- 0 . 2 1}$ & 0.00 \\
\hline Conflicting Speed & & & & & & & & -0.07 \\
\hline
\end{tabular}




\section{(3) Effect of significant variables}

In the forward stepwise logistic regression analysis process, each significant variable was entered into the model according to its p-value. Table 3 presents the results of the stepwise analysis, including the variables at each step and parameters estimation. The effect of each variable, entering at each step, was described in the following sub-sections.

\section{a) Gap size}

The relationship between the gap size and probability of accepting the gap was shown in Fig. 2. At the $\mathrm{P}$ (accept) of 0.5 , the gap size was 5.0 seconds. This implied that when a u-turning vehicle faced a gap size of greater than or equal to 5.0 seconds, the vehicle would accept the gap and make u-turn.

\section{b) Speed of conflicting vehicle}

The speed of the conflicting vehicle affected the gap acceptance behavior. As shown in Fig. 3, the higher speed, the less probability of accepting the gap for the same gap size. At the $\mathrm{P}$ (accept) of 0.5 , the gap

Table 2 Significant test of all variables.

\begin{tabular}{lrrr}
\hline Variable & Wald's & df & p-value \\
\hline Gender & 0.84 & 1 & 0.36 \\
Age & 1.41 & 2 & 0.49 \\
U-turn Veh. Type & 1.08 & 2 & 0.58 \\
Queue Time & 0.00 & 1 & 0.99 \\
Wait Time & 23.15 & 1 & 0.00 \\
Gap Size & 113.82 & 1 & 0.00 \\
Conflicting Speed & 36.69 & 1 & 0.00 \\
Conflict Veh. Type & 4.87 & 3 & 0.18 \\
U-turn Veh.Type $\times$ & 8.14 & 6 & 0.23 \\
Conflict Veh.Type & & & \\
\hline Goodness-of-fit Test & $\chi^{2}$ & df & p-value \\
\hline Hosmer and Lemeshow & 13.51 & 8 & 0.10 \\
\hline
\end{tabular}

Note: $d f=$ degree of freedom size was 4.0, 5.0, and 6.0 seconds for the conflicting vehicle's speed of 30,50 , and $70 \mathrm{~km} / \mathrm{hr}$, respectively.

\section{c) Wait time}

The wait time of the u-turning vehicle affected the gap acceptance behavior. As shown in Fig. 4, the longer wait time, the higher probability of accepting the gap for the same gap size. At the P(accept) of 0.5 , the gap size was 5.6, 5.0, 4.5 and 4.0 seconds for the wait time of $0,15,30$, and 45 seconds, respectively. This was based on the conflicting speed of $50 \mathrm{~km} / \mathrm{hr}$.

\section{d) Correlation among variables}

The correlation matrix from the forward stepwise analysis was shown in Table 4. The gap size and conflicting speed seemed to have some negative relationship, but not so high. Therefore, it was better to leave both variables in the model to yield the more accurate prediction results. In addition, the wait time seemed to have no correlation with gap size and conflicting speed.

\section{(4) Decision model}

To predict the u-turn decision at the confidence interval of $95 \%$, three variables were included in the model formulation. The developed u-turn decision model was shown as follows:

$$
\begin{gathered}
\mathrm{P}(\text { accept })=\frac{e^{z}}{1+e^{z}}=\frac{1}{1+e^{-z}} \\
z=-3.802+1.235 \mathrm{Gap}-0.062 \text { Speed }+0.043 \text { Wait }(2 \mathrm{~b})
\end{gathered}
$$

The model performance was shown in Table 5 . The model yielded the Nagelkerke $\mathrm{R}^{2}$ of 0.69 . The Hosmer and Lemeshow test also indicated the goodness-of-fit of the observed and predicted events ( $p$-value $>0.05$ ).

The classification table showing the percentage correctness of the model prediction was shown in Table 6 for both the cases that were used and not used in the model development process.

Table 3 Forward stepwise regression analysis result.

\begin{tabular}{llrrrrrc}
\hline & Variable & B & S.E. & Wald's $\chi^{2}$ & df & p-value & $\operatorname{Exp}(\mathrm{B})$ \\
\hline \multirow{2}{*}{ Step 1 } & Constant & -6.129 & 0.502 & 149.15 & 1 & 0.00 & 0.002 \\
& Gap Size & 1.221 & 0.101 & 145.09 & 1 & 0.00 & 3.391 \\
\hline \multirow{2}{*}{ Step 2 } & Constant & -3.107 & 0.627 & 24.56 & 1 & 0.00 & 0.045 \\
& Gap Size & 1.270 & 0.111 & 131.51 & 1 & 0.00 & 3.560 \\
& Conflicting Speed & -0.064 & 0.010 & 41.14 & 1 & 0.00 & 0.938 \\
\hline \multirow{2}{*}{ Step 3 } & Constant & -3.802 & 0.665 & 32.66 & 1 & 0.00 & 0.022 \\
& Gap Size & 1.235 & 0.112 & 122.59 & 1 & 0.00 & 3.439 \\
& Conflicting Speed & -0.062 & 0.010 & 35.48 & 1 & 0.00 & 0.940 \\
& Wait Time & 0.043 & 0.010 & 19.83 & 1 & 0.00 & 1.044 \\
\hline
\end{tabular}

Note: $B=$ Estimated Parameter, S.E. $=$ Standard Error, $d f=$ degree of freedom 


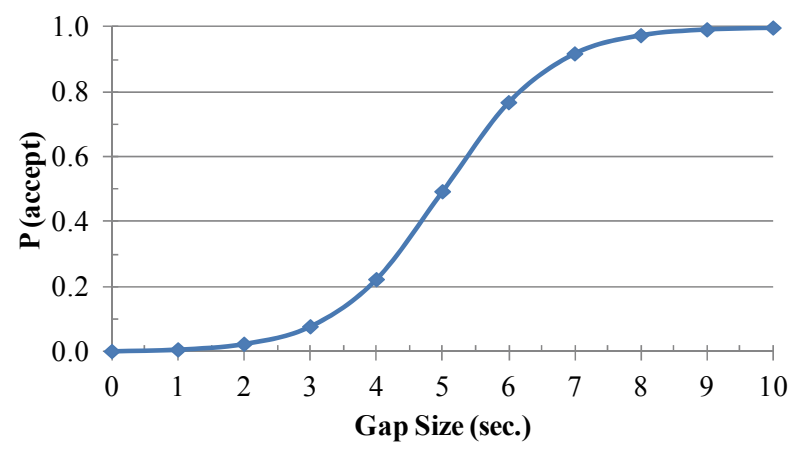

Fig. 2 Effect of gap size on u-turn decision.

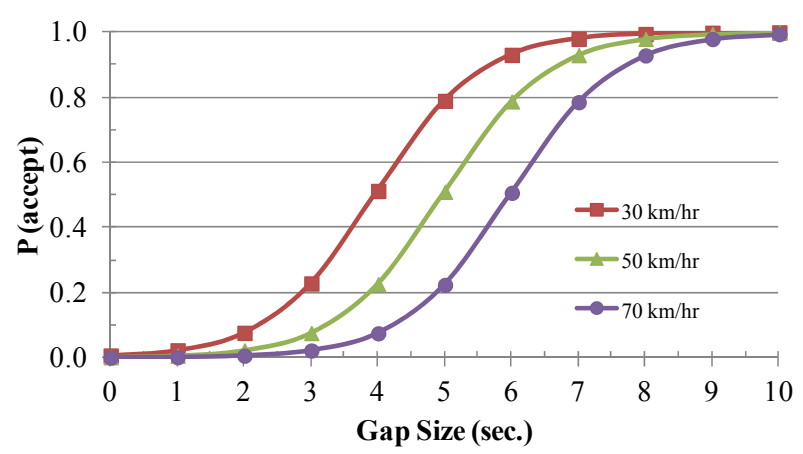

Fig. 3 Effect of conflicting vehicle speed on u-turn decision.

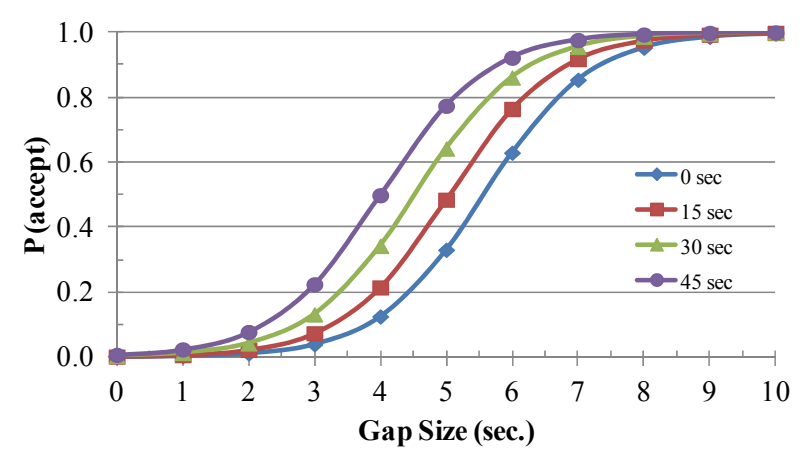

Note: Assume the conflicting speed of $50 \mathrm{~km} / \mathrm{hr}$

Fig. 4 Effect of wait time on u-turn decision.

Table 4 Correlation matrix from the stepwise analysis.

\begin{tabular}{llccc}
\hline & & Constant & Gap Size & Speed \\
\hline Step 1 & Gap Size & -0.970 & & \\
\hline Step 2 & Gap Size & -0.616 & & \\
& Speed & -0.550 & -0.288 & \\
\hline \multirow{2}{*}{ Step 3 } & Gap Size & -0.598 & & \\
& Speed & -0.520 & -0.303 & \\
& Wait Time & -0.268 & 0.069 & -0.040 \\
\hline
\end{tabular}

Table 5 Model performance.

\begin{tabular}{crrr}
\hline Goodness-of-fit Test & $\chi^{2}$ & df & p-value \\
\hline Hosmer and Lemeshow & 7.78 & 8 & 0.46 \\
\hline \multicolumn{3}{c}{ Model R } \\
\hline Cox \& Snell R \\
Nagelkerke R ${ }^{2}$ & 0.52 & \\
\hline
\end{tabular}

Table 6 Classification table showing the prediction result.

\begin{tabular}{|c|c|c|c|c|}
\hline & \multirow{2}{*}{\multicolumn{3}{|c|}{$\frac{\text { Predicted }}{\text { Cases for model development }}$}} \\
\hline \multirow{10}{*}{$\begin{array}{l}\vec{D} \\
\sum_{0}^{0} \\
0 \\
0\end{array}$} & \multirow{5}{*}{$\begin{array}{l}\text { Rejected } \\
\text { Accepted }\end{array}$} & & & \\
\hline & & Rejected & Accepted & $\%$ Correct \\
\hline & & 239 & 38 & 86.28 \\
\hline & & 39 & 214 & 84.58 \\
\hline & & Overall $\mathrm{P}$ & rcentage & 85.47 \\
\hline & \multirow{5}{*}{$\begin{array}{l}\text { Rejected } \\
\text { Accepted }\end{array}$} & \multicolumn{3}{|c|}{ Cases for model validation } \\
\hline & & Rejected & Accepted & $\%$ Correct \\
\hline & & 25 & 4 & 86.21 \\
\hline & & 4 & 30 & 88.24 \\
\hline & & \multicolumn{2}{|c|}{ Overall Percentage } & 87.30 \\
\hline
\end{tabular}

\section{DISCUSSIONS AND CONCLUSIONS}

The findings from this research could illustrate the distinctive characteristics of u-turn gap acceptance behavior. For TWSC intersection, the mean accepted gap tends to decrease as the queue time or wait time increases ${ }^{5}$. From the result of this research, the queue time was not statistically significant for the u-turn gap acceptance. The u-turn vehicles, when staying in queue, could see the conflicting traffic stream and realize the traffic situation. Therefore, the u-turn drivers would not take the delay in queue to decide whether or not accepting the gap. In other words, the waiting of u-turn drivers might be memoryless. Nevertheless, it also depended on the nature of the driver population in the area.

Unlike the past research ${ }^{9}$, the results of this study showed that the conflicting speed and wait time also influenced the u-turn decision. The past study indicated the significance of wait time in the morning and noon periods, but not in the afternoon period. Thus, the wait time was not selected due to its inconsistency. Nevertheless, the wait time might be significant in the afternoon period on the other days. In addition, the different site characteristics might differ the analysis results. The presence of crosswalk might cause the insignificance of conflicting speed in u-turn decision. Instead, the u-turn vehicle might consider whether the conflicting vehicle was accelerating or decelerating.

Some factors affecting the u-turn gap acceptance 
and aggressive behaviors were different. Conflicting speed was the same factor affecting both behaviors. The aggressive behavior is mainly the outcome of attitudinal characters of driver, not dependent on driver's wait time ${ }^{10)}$. Most aggressive drivers (90\%) conducted the forcing maneuver after waiting not more than 10 seconds. However, based on the result of the present study, when the wait time was as high as 45 seconds, the driver would accept the gap as low as 4.0 seconds, which was less than the critical gap of 4.3 seconds ${ }^{11)}$. Thus, the longer waiting time would contribute to the unsafe movement, implying that the driver himself is not an aggressive driver but could induce an aggressive behavior. On the contrary, the real aggressive driver would conduct the forcing maneuver at the beginning of his waiting, without considering the wait time.

The conclusions could be listed as follows:

- gap size, conflicting speed, and wait time significantly affected the u-turn decision at $95 \%$ confidence interval;

- gap size influenced the most to the u-turn decision, followed by conflicting speed and wait time, respectively;

- u-turn driver age and gender, vehicle type of both u-turning and conflicting traffic, and queue time did not significantly influence the decision at $95 \%$ confidence interval;

- u-turn decision model based on gap size, speed and wait time could predict well with the percentage correctness of more than $85 \%$.

The factors affecting the u-turn decision at midblock median opening were addressed in this paper. Each significant factor could be investigated in the further studies. The relationships between conflicting traffic characteristics (e.g., volume, headway, etc.) and gap size and/or speed can be examined. In addition, the wait time of u-turn vehicle may have relationships with conflicting traffic characteristics. The further studies will provide a clear understanding on the traffic operation at the u-turn junction, leading to the better traffic management. The future research can also focus on the control and management at such a median opening to ensure the traffic flow and safety. Some proposed improvement schemes can be studied in detail to evaluate the efficiency and effectiveness.

\section{REFERENCES}

1) Transportation Research Board, Highway Capacity Manual, $5^{\text {th }}$ Edition, National Research Council, USA, 2010.

2) Liu, P., Lu, J. J. and Cao, B. : Capacity of u-turns at unsignalized median openings on six-lane streets, Transportation Research Record, No. 2130, pp. 59-65, 2009.

3) Dissanayake, S., Lu, J. J. and Yi, P. : Driver age differences in day and night gap acceptance capabilities, IATSS Research, Vol. 26, No. 1, pp. 71-79, 2002.

4) Yan, X., Radwan, E. and Guo, D. : Effects of major-road vehicle speed and driver age and gender on left-turn gap acceptance, Accident Analysis and Prevention, No. 39, pp. 843-852, 2007.

5) Kyte, M., Clemow, C., Mahfood, N., Lall, B. K. and Khisty, C. J. : Capacity and delay characteristics of two-way stop-controlled intersections, Transportation Research Record, No. 1320, pp. 160-167, 1991.

6) Pollatschek, M. A., Polus, A. and Livneh, M. : A decision model for gap acceptance and capacity at intersections, Transportation Research Part B, Vol. 36, Issue 7, pp. 649-663, 2002.

7) Combinido, J. S. L. and Lim, M. T. : Modeling U-turn traffic flow, Physica A, Vol. 389, Issue 17, pp. 3640-3647, 2010.

8) Pirdavani, A., Brijs, T., Bellemans, T. and Wets, G. : Travel time evaluation of a u-turn facility: comparison with a conventional signalized intersection, Transportation Research Record, No. 2223, pp. 26-33, 2011.

9) Ebisawa, R., Espada, I. C., Nakatsuji, T. and Tanaboriboon, Y. : Gap acceptance characteristics at a u-turn section in Bangkok, J. of the Eastern Asia Society for Transportation Studies, Vol. 4, No. 2, pp. 63-74, 2001.

10) Kaysi, I. A. and Abbany, A. S. : Modeling aggressive driver behavior at unsignalized intersections, Accident Analysis and Prevention, Vol. 39, pp. 671-678, 2007.

11) Jenjiwattanakul, T. and Sano, K. : Effect of waiting time on the gap acceptance behavior of $\mathrm{u}$-turning vehicles at midblock median openings, J. of the Eastern Asia Society for Transportation Studies, Vol. 9, pp. 1601-1613, 2011.

(Received February 25, 2012) 